\title{
Factor of Determination of the Center of Gravity Point on Sitting of the Right and Left Axis in Patients with Hemiplegia
}

\author{
Shu Morioka ${ }^{1)}$, Midori Hidaka ${ }^{2)}$, Shozo Miyamoto ${ }^{1)}$ \\ ${ }^{1)}$ Department of Physical Therapy, Kochi School of Allied Health and Medical Professions, \\ 6012-1, Nagahama, Kochi 781-0270, Japan. TEL +81 88-842-0412 \\ ${ }^{2)}$ Department of Rehabilitation, Atago Hospital
}

\begin{abstract}
The purpose of this study was to investigate the factor of determination of the center of gravity of the right and left axis in sitting of hemiplegic patients. Subjects were 30 hemiplegic patients. The subjects center of gravity point was measured during 1) natural sitting, 2) sitting on the non-paretic weight-bearing, and 3) sitting on the paretic weight-bearing under eyes open and eyes closed conditions for ten seconds. We calculated the middle point value for 2) and 3), and observed a correlation between the center of gravity point during natural sitting and the middle point. This result indicate that the center of gravity point is decided by relative position of the maximal voluntary weight-bearing of the nonparetic and paretic sides.
\end{abstract}

Key words: Center of gravity point, Sitting, Hemiplegia.

(This article was submitted Jul. 19, 1999, and was accepted Sep. 14, 1999)

\section{INTRODUCTION}

The center of gravity of right and left in sitting of hemiplegic patients depends on the weight of the non paretic side center of gravity bearing on the buttocks. This phenomenon affects trunk function and a degree is decided for the buttocks of the paretic side ${ }^{1)}$.

Recently, there have been reports about the center of gravity point from the view-point of the center of pressure on the buttocks by spread using a center of gravity recorder ${ }^{2-4)}$. An index of analysis on sitting for hemiplegic patients using the center of gravity recorder parameters found not only the amounts of sway from length and area but also the center of pressure divided on the right and left axis or the front and rear axis during the recording time. Generally, the center of gravity point in two-dimensions at the center of pressure point may well be made a value of center of gravity sway at a locus value of movement of the center of pressure. In particular, a measurement of the center of gravity point is important because then we have knowledge of the rate of weight bearing on the buttocks of hemiplegic patients.

We clarified the center of gravity point in standing of hemiplegic patients which was decided for the relative point on the center of foot pressure at maximal voluntary weight-bearing for the nonparetic side and the paretic side ${ }^{5}$. The purpose of this study was to investigate that hypothesis regarding the sitting position.

\section{SUBJECTS}

Thirty hemiplegic patients who could maintain a sitting position for a long time participated in this study. Right hemiplegia were 14 subjects, and left hemiplegia were 16 subjects. Their mean age was $62.3 \pm 14.2$ (mean \pm standard deviation) years old. 
All subjects had no previous record of higher cortical dysfunction.

\section{METHODS}

A center of gravity recorder (gravicorder GS2000, ANIMA. CO.) was used in this study. The subjects center of gravity point was measured under three conditions: 1) natural sitting, 2) maintained sitting of maximal voluntary weight-bearing on the non paretic side (sitting on the non-paretic weight-bearing), and 3) maintained sitting of maximal voluntary weight-bearing on the paretic side (sitting on the paretic weight-bearing) with eyes open and eyes closed for ten seconds. In addition, the feet were hanging above the floor during sitting. The parameter used was the mean of deviation $\mathrm{X}$ (the right and left) axis.

Mean and standard deviation for each item analyzed were calculated. Also, the middle point was calculated from the non-paretic weight-bearing point and the paretic weight-bearing point in the eyes open and closed conditions (Fig. 1). The non-

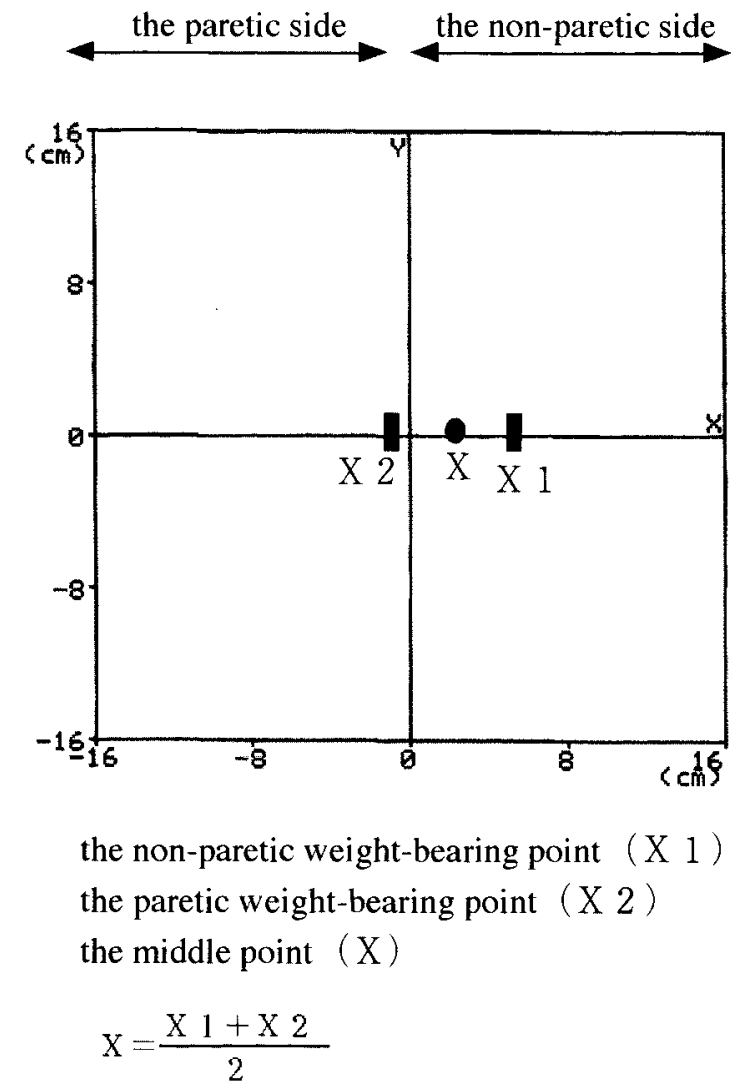

Fig. 1. The calculation method of the middle point. paretic side was indicated plus, and the paretic was indicated minus in order to unite of the non-paretic side and the paretic side (the right and left). Pearson's product moment correlation coefficient was calculated between the center of gravity point in natural sitting and the middle point.

\section{RESULTS}

The average value of the center of gravity point of natural sitting was $0.28 \pm 1.12 \mathrm{~cm}$, the nonparetic weight-bearing point was $4.21 \pm 1.21 \mathrm{~cm}$, the paretic weight-bearing point was $3.87 \pm 1.85$ $\mathrm{cm}$, and the middle point was $0.17 \pm 1.14 \mathrm{~cm}$ in the open eyes condition. The correlation coefficient was $0.84(p<0.01)$ between the center of gravity point of natural sitting and the middle point (Fig. 2-a).

The average value of the center of gravity point on natural sitting was $-0.17 \pm 1.15 \mathrm{~cm}$, the nonparetic weight-bearing point was $3.52 \pm 1.11 \mathrm{~cm}$, the paretic weight-bearing point was $3.62 \pm 1.44$ $\mathrm{cm}$, and the middle point was $-0.05 \pm 0.94 \mathrm{~cm}$ in the closed eyes condition. The correlation coefficient was $0.89(\mathrm{p}<0.01)$ between the center of gravity point of natural sitting and the middle point (Fig. 2-b).

\section{DISCUSSION}

The center of gravity point of the right and left of the amount of weight-bearing on the right and left sides, and the center of pressure point, were analyzed. Previous reports have indicated that hemiplegic patients were partial to the non-paretic side as regards the center of gravity point ${ }^{6-9)}$. Because there is dysfunction of motor and sense on the paretic side, the dysfuction of the trunk ${ }^{1)}$ and a decrease in weight distribution from the dysfunction paretic side and trunk was indicated.

Generally, the center of gravity point in sitting of the right and left axis is measured at the center of the buttocks using the center of gravity recorder. Therefore, a value of the mean of deviation for the $\mathrm{X}$ (right and left) axis is the center of gravity point at the right and left axis. This time we investigated the relationship between the center of gravity point at the right and left axis and the middle point. The middle point is calculated as the non-paretic weight-bearing point and the paretic weight-bearing point. This middle point is located at the point 

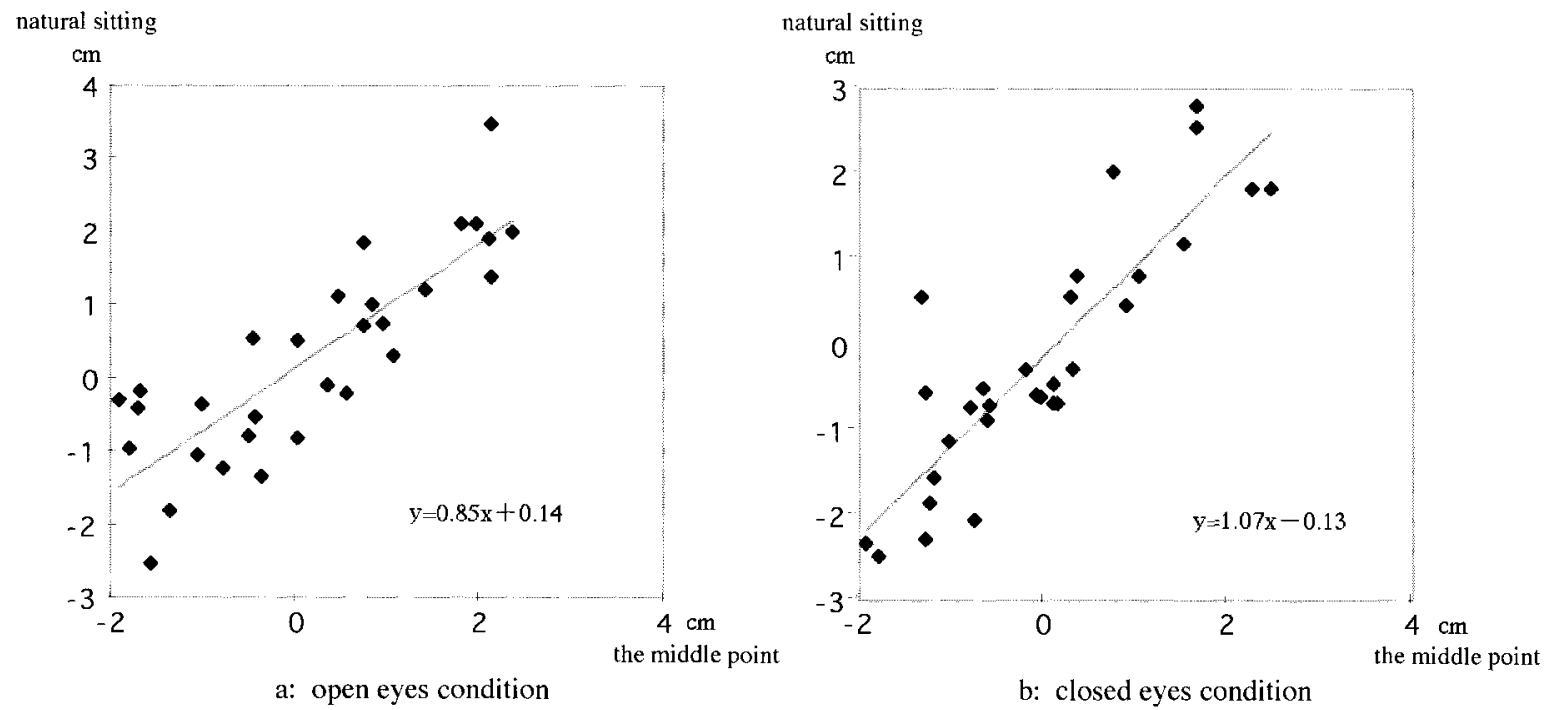

Fig. 2. The relationship between the center of gravity point at natural sitting and the middle point.

of intersection of the right and left axis and the front and rear axis when the non-paretic weightbearing width is equal to the paretic weight-bearing width.

We have reported the correlation between the center of gravity point on the right and left axis in sitting and the middle point in standing ${ }^{5)}$. This result indicates that the center of gravity point on the right and left axis in sitting of hemiplegic patients is not only a trend of weight-shifting on the non-paretic side, affected as a result of the paretic side, but also that the center of gravity point on the right and left axis in sitting is in the habit of occurring at the center position of a point of sitting on the non-paretic weight-bearing side and a point of sitting on the paretic weight-bearing side. In addition, the center of gravity point of the right and left axis in sitting is formed by the reciprocal action of the environment and the patient after the onset of disease in hemiplegia. Therefore, the center of gravity point on the right and left axis in sitting of hemiplegic patients is decided by the relative point of the center of buttocks pressure at maximal voluntary weight-bearing for the non-paretic side and the paretic side. Further studies are required to investigate the differences between the eyes open and eyes closed conditions.

\section{REFERENCES}

1) Tomita M, Sato F, Uno J, et al.: Trunk movement; trunk movement in hemiplegia. Rigaku ryoho janaru, 1991, 25: 88-94.

2) Stadin KJ, Smith BS: The measure of balance in sitting in stroke rehabilitation prognosis, Stroke, 1990, 21: 82-86.

3) Takahashi T, Amimoto K, Sugimoto S, et al.: Prediction of the outcome of gait prognosis clinical significance on dynamic sitting balance test in acute stroke. Rigakuryohogaku, 1992, 19: 592-596.

4) Uchiyama $Y$, Minejima T, Aruga T, et al.: Postural regulability on the sitting position and its application to physical therapy field. Rigakuryohogaku, 1994, 21: 179-185.

5) Hidaka M, Morioka S, Miyamoto S, et al.: Factor of determination of the center of gravity point on standing in patients with hemiplegia. Rigakuryohogaku, 1998, 25 (supple): 412.

6) Arcan M, Brull MA, Najenson T, et al.: FGP assessment of postural disorders during process of rehabilitation. Scand J Rehabil Med, 1977, 9: 165168.

7) Bohannon RW, Larkin PA: Lower extremity weight bearing under various standing conditions in independently ambulatory patients with hemiparesis. Phys Ther, 1985, 65: 1323-1325.

8) Dickstein R, Nissan M, Pillar T, et al.: Foot-ground pressure pattern of standing hemiplegic patients. Phys Ther, 1984, 64: 19-23.

9) Dettmann MA, Linder MT, Sepic SB, et al.: Relationships among walking performance, postural stability, and functional assessments of the hemiplegic patients. Am J Phys Med, 1987, 66: 77-90. 